\title{
Tuberculosis in South Asia
}

\author{
Rakesh Kumar and Shalini Singh* \\ QSAR \& Cheminformatics Laboratory, Department of Chemistry, Bareilly College, India
}

*Corresponding author: Shalini Singh, QSAR \& Cheminformatics Laboratory, Department of Chemistry, Bareilly College, Bareilly, UP, India

\begin{tabular}{|c|c|}
\hline ARTICLE INFO & ABSTRACT \\
\hline Received: 豐 December 14, 2021 & Keywords: Rifampicin; Bedaquiline; Mycobacterium Tuberculosis \\
\hline Published: 㓞 December 22, 2021 & $\begin{array}{l}\text { Abbrevations: TB: Tuberculosis; SEA: South-East Asia; MDR: Multidrug Resistant } \\
\text { Tuberculosis; IPT: Isoniazid Preventive Therapy; GCP: Good Clinical Practice }\end{array}$ \\
\hline $\begin{array}{l}\text { Citation: Rakesh Kumar, Shalini Sin- } \\
\text { gh. Tuberculosis in South Asia. Biomed } \\
\text { J Sci \& Tech Res 40(4)-2021. BJSTR. } \\
\text { MS.ID.006496. }\end{array}$ & \\
\hline
\end{tabular}

\section{Introduction}

Tuberculosis (TB) is a potentially serious infectious disease that mainly affects the lungs though it can spread to other parts of the body one's brain or spine. The bacteria are spread when an infected person coughs or sneezes. It can also spread through saliva (by kissing or sharing drinks etc.). A type of bacteria called Mycobacterium tuberculosis is the cause of the disease. Tuberculosis (TB) remains formidable threat to global health despite concerted efforts during past decades by the global health community. From 1990 to 2015 the death rate has decreased by 47 percent [1]. Among 22 countries which had high TB burden, 16 countries have achieved millennium development goals for TB. A target has been set by the Global Health Community for the post 2015, ENO-TB strategy of reduction of 25 percent in occurrence and a reduction of 75 percent in mortality between 2015 and 2025 and a mortality reduction of 95 percent and 90 percent reduction in occurrence by the year 2035 [1]. As per the news item published in the daily, "The Times of India" dated 16th February 2020, The Health Minister of India, Dr. Harsh Vardhan informed that the country has even more ambitious target of eliminating the disease from the country by the year, 2025 and said that the mission Indradhanush is already operational for the purpose.

Although the elimination of a disease like TB is a very complex problem but by the time our understanding and knowledge about infectious diseases, global connections, resources and range of intercession have also increased. In order to increase the rate of TB elimination we must increase the research to maximum level in high burden countries like India, Pakistan, Nepal, Bangladesh, Afghanistan, Maldives, Bhutan and Sri Lanka. The WHO South-East Asia (SEA) is habitat for $26 \%$ of the world's population with $44 \%$ heavy load of TB. An estimated 4.4 million community got sick with TB and estimated 6,38,000 passed away due to the disease which is over half of the global TB deaths [2]. The coming into view of rifampicin resistant or multidrug resistant tuberculosis (MDR) is one of the most important challenges to the control of tuberculosis pandemic. The main reason for drug resistant tuberculosis is either the transmission of already resistant strain of Mycobacterium tuberculosis or the suboptimal cure of susceptible strains. It is no surprise therefore that having such a high burden of TB disease, this region has an estimated number of 184336 MDR cases among the total recorded TB cases which brings it on third position in the list of Global MDR burden region [2]. Most of the MDR cases in this field remain untouched due to considerable gap between the 
agreement and useful putting into effect [3]. As such, there is an imperative need to improve and strengthen research capacity in these high burden countries.

\section{Treatment}

In our fight against drug resistant $\mathrm{TB}$, a great achievement is the recent licensing of bedaquiline and delamanid [4-5]. Effective treatment depends upon accurate diagnosis. In the region, the Gene X port scale up has significantly increased detection of MDR TB [6]. Until there is an improvement in molecular drug susceptibility testing for drugs other than rifampicin, phenotype drug susceptibility testing will remain essential to treat MDR TB cases properly. Although the population is very large (1.8 billion), the ratio of DST laboratories to population remains as low as 0.2 labs per $5 \mathrm{M}$ population. It is estimated that the cost of treatment of a single MDR patient is about 500 times the cost of a drug susceptible patient. Therefore investment in strong MDR TB control is very cost effective if it successfully checks MDR transmission. Apart from this, the new shorter MDR TB treatment regimen from Bangladesh is now adopted and recommended by WHO and it should also be implemented region wide.

\section{Infection Control and their Prevention}

The huge number of latently infected individuals throughout the world presents an extensive challenge to eradication efforts. Various models created for control strategies suggested that TB suppression is not possible without get to grips with latent TB. A rough estimate was that about $1 / 3^{\text {rd }}$ of the world population was suffering from latent TB. But according to a recent study the risk of infection per year estimates around one fourth of the total world population which counts near 1.7B [7]. In the last decade, Isoniazid preventive therapy (IPT) in endemic countries has received serious consideration. Shorter regimens must be developed for its large scale feasibility also the regimens appropriate in the area of high isoniazid resistance. The 12 doses given 3 months rifapentine isoniazid regimine is a step in the right direction but more must be done to value carefully worked designs for IPT scaleup beyond those currently being undertaken in people living with HIV. South Asia is also vulnerable to natural disasters and political un stability. Contingency measures are to be kept in place for prevention of decease spread in case a disaster visits. The 2015 earthquake in Nepal and lack of proper delivery system of healthcare measures is a grim reminder in this regard.

\section{Research Priorities}

The discovery of an effective vaccine to treat TB seems an unachievable goal in the near future. While efforts must be kept on for finding a vaccine, we should find a way to eradicate TB without using any vaccine. In large urban cities and remote rural areas of south Asian region research priorities must aim at packaged intrusions. Research capacity should be increased by broadening the network of good clinical practice (GCP) compliant, clinical trial sites to spread up novel regimen evaluations, improving laboratory capacity for bacterial culture and testing of drug susceptibility. Networking research wideness the region to share and scale up best experiences, enthralling governments and fund providers in research plans to make certain move and political promise to keep giving money (Figure 1).

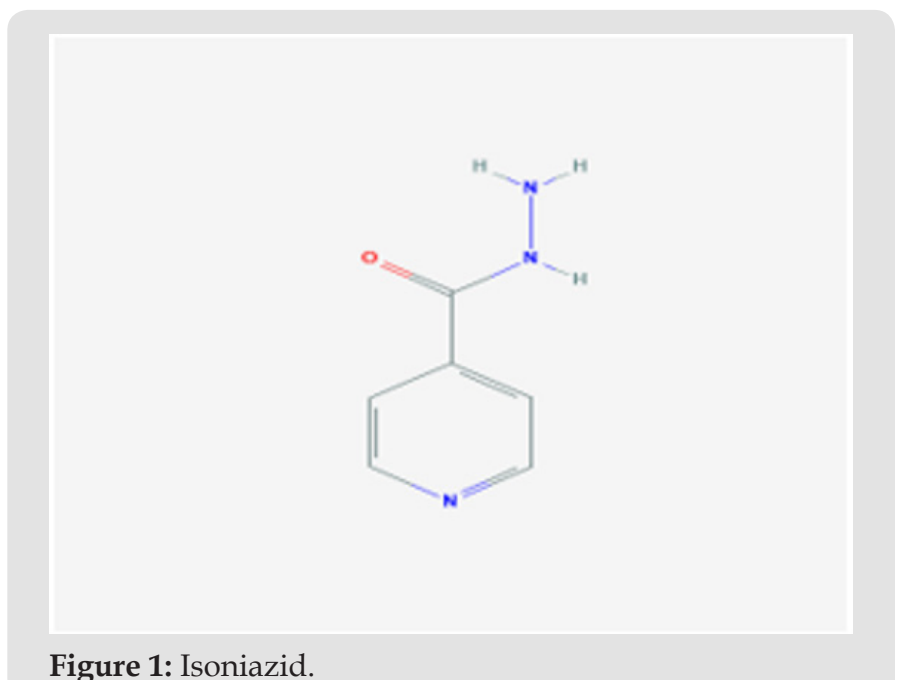

\section{Conclusion}

There is an golden chance for the scientific fraternity to embark on a path of new research to find real cure for the huge majority of TB afflicted population in the South Asian region. South Asia has patients with a large number of innovative scientists who can enable it to seize the opportunity and lead global TB eradication programs by representing feasibility in cities with high population density and remotest reaches of the Himalayas. Political will and leadership with a vision are the two attributes which are essential to facilitate the loftily programme of TB elimination. The malady cannot be overcome by a single intervention, instead continuous innovations are required. This is the time that researchers in the region join hands, work with cohesion, develop an unified agendum to ensure successful research for TB eradication. The sooner, the better.

\section{References}

1. (2018) WHO. World Health Organisation END TB strategy, 2015.

2. (2018) WHO. World Health Organisation. Global tuberculosis Report 2017.

3. (2017) Medecins sans Frontiers/STOP-TB partnership. Out of Step: TB policies in 29 countries.

4. Dheda K, Barry CE, Maartens G (2016) Tuberculosis. Lancet 387(10024): 1211-1226. 
5. Udwadia ZF, Amale RA, Mullerpattan JB (2014) Initial experience of bedaquiline use in a series of drug-resistant tuberculosis patients from India. Int J Tuberc Lung Dis 18(11): 1315-1318.

6. Sachdeva KS, Raizada N, Sreenivas A, Vant Hoog AH, Van den Hof S, et al. (2015) Use of Xpert MTB/RIF in decentralized public health settings and its effect on pulmonary TB and DR-TB case finding in India. PLoS One 10(5): e0126065.

ISSN: 2574-1241

DOI: 10.26717/BJSTR.2021.40.006496

Shalini Singh. Biomed J Sci \& Tech Res

(C) (P) This work is licensed under Creative

Submission Link: https://biomedres.us/submit-manuscript.php
7. Houben RM, Dodd PJ (2016) The global burden of latent tuberculosis infection: a re-estimation using mathematical modelling. PLoS Med 13(10): e1002152.

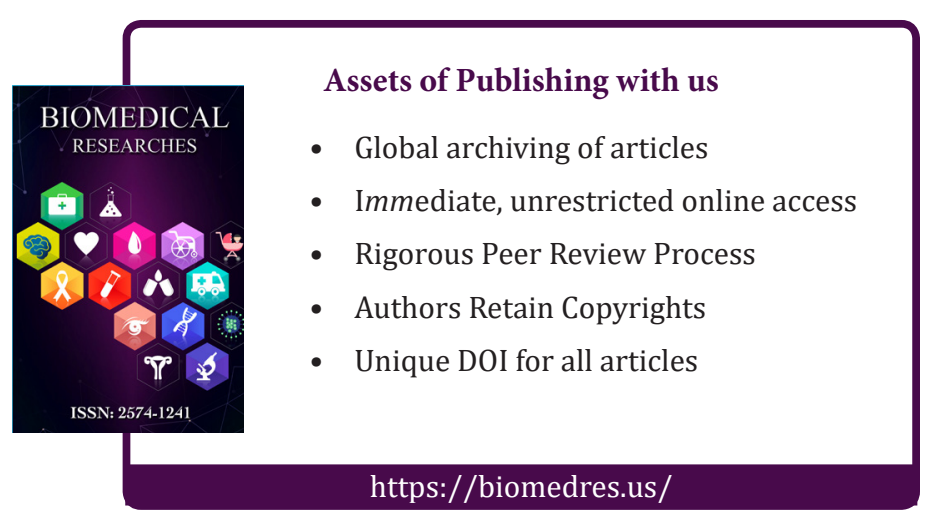

